\title{
Contactless Detection of Heartbeat and Cardiopulmonary Modeling using Vector Analyzer
}

\author{
M. Raja, S. Dhanasekaran, C. Bala Subramanian
}

\begin{abstract}
Now a days, Modern world makes it difficult for some individuals to care for their health. Urban air pollution, employment pressure, and an uneven diet increase a person's likelihood of being infected. In practice, until serious things, some of the infections would not provoke any symptoms. Heart rate $(\mathrm{HR})$ is a measure of physiological activity. This article introduces contactless heartbeat detection and cardiopulmonary modeling. Our suggested microwave system uses a vector network analyzer to demonstrate the potential to detect the heartbeat signal at distinguishable frequency ranges and at distinct output energy concentrations. The model comprising the heartbeat and breathing signals are provided based on variables obtained from actual measurements. To separate the heartbeat and breathing signals, various processing methods are used. For separate signal-to noise ratios, wavelet filters possess greater accuracy over standard filters in order to determine heart rate and heart rate variation.
\end{abstract}

Keywords: coronary heart disease, Ultra-Wideband (UWB) radar, Doppler hypothesis

\section{INTRODUCTION}

For patients with circumstances, electrocardiogram s are uncomfortable. for example, consume unfortunate casualties or recently conceived babies. In light of the Doppler hypothesis, an objective with a semi intermittent development mirrors the transmitted flag with its stage adjusted when shifting position of the objective. Henceforth, the reflected flag off the individual's chest contains data about the chest dislodging, because of heartbeat and breath. Then again, while holding breath, the thought about flag depends the chest relocation because of heartbeat alone. The chest relocation variety, brought about by breath, is somewhere in the range of 4 and $12 \mathrm{~mm}$. Be that as it may, the chest dislodging because of heartbeat alone ranges somewhere in the range of 0.2 and $0.5 \mathrm{~mm}$. Additionally very still, the breath rate compares to a recurrence that differs somewhere in the range of 0.1 and $0.3 \mathrm{~Hz}$, while the heartbeat rate relates to a recurrence that fluctuates inside the $1-3 \mathrm{~Hz}$ interim. Related work was expected to distinguish life signs, breath rates and heartbeat rates, utilizing fixed recurrence and fixed intensity of the transmitted flag. Direct- transformation Doppler radars have been integrated into $0.25 \mu \mathrm{m}$ CMOS and BiCMOS techniques working at $1.6 \mathrm{G}$.

Revised Manuscript Received on December 09, 2019

M.Raja, Department of Computer Science and Engineering, Kalaslingam Academy of Research and Education, Krishnankoil, Tamil Nadu, India. kingraaja@gmail.com

Dr. S. Dhanasekaran, Department of Computer Science and Engineering, Kalaslingam Academy of Research and Education, Krishnankoil, Tamil Nadu, India. srividhans@ gmail.com

Mr. C. Bala Subramanian, Department of Computer Science and Engineering, Kalaslingam Academy of Research and Education, Krishnankoil, Tamil Nadu, India. kingraaja@gmail.com

\section{THEORITICAL BACKGROUND}

Heartbeat and breath signals are demonstrated with direct capacities; for example, each heartbeat (beat or breath) is displayed with two connected direct capacities. The proposed model considers three fundamental highlights. The main component is to safeguard the amplitudes of the deliberate signs.

This ensures a steady proportion between the abundancy of the heartbeat flag and the sufficiency of the breath flag. The second consideration is the heartbeat rate. This variable is removed from the firstflag and is recognized as a source of view on which the se parated flag's heartbeat rate is perceived. The third factor i s the variability of the pulse. A white Gaussian clamor is added to the entirety of the heartbeat flag and the breath flag. The commotion tests are produced as pseudo- arbitrary qualities drawn from an ordinary circulation with mean zero and standard deviation one $(1=n \sigma)$. The amplitudes of the heartbeat and the breath signals are diminished by a similar proportion so as to have diverse SNR levels. At that point the partition of the heartbeat and the breath signals is prepared utilizing distinctive procedures at each SNR level. The SNR esteems are set among 0 and $-20 \mathrm{~dB}$ by a stage of $1 \mathrm{~dB}$.

\section{PROBLEM DEFINITION}

In this theory we will reproduce the technique created by Poh et al. (2010) for estimating pulse with a minimal effort camera and just encompassing light, in the expectation of accomplishing comparative outcomes. We will inspect if the decision of shading space has any influence on the outcomes. Besides, we will take a gander at options to ICA for separating the pulse. We will take a gander at the mistake presented by shortening the length of the video succession utilized for the estimation. Thiswill be utilized to decide an adequate length for use in conceivable applications. Also, we expect to develop this technique so as to make a HRIM of the face to demonstrate where the pulse is generally unmistakable. This can be utilized to identify variations from the norm in the facial blood flow. The new learning about the HRIM can be utilized to improve the discovery of pulse in common recordings. Utilizing the pulse estimation strategy, we need to look at the connection between the time difference of when blood from a heartbeat achieves the hand and face and the circulatory strain.

\section{EXISTING SYSTEM}

The usefulness of the Doppler microwave radar used in ho me checking has extended late. For patients with circumstances, electrocardiograms are annoying, for example, consume exploited people or recently conceived newborn children. 


\section{Contactless Detection of Heartbeat and Cardiopulmonary Modeling using Vector Analyzer}

In light of the Doppler hypothesis, an objective with a semi occasional development mirrors the transmitted flag with its stage regulated by the time varying position of the objective. Consequently, the reflected flag off the individual's chest contains data about the chest uprooting, because of heartbeat and breath. Then again, while holding breath, the considered flag depends the chest relocation because of heartbeat alone. The chest dislodging variety, brought about by breath, is somewhere in the range of 4 and $12 \mathrm{~mm}$. In any case, the chest removal because of heartbeat alone ranges somewhere in the range of 0.2 and $0.5 \mathrm{~mm}$. Likewise very still, the breath rate compares to a recurrence that changes somewhere in the range of 0.1 and $0.3 \mathrm{~Hz}$, while the heartbeat rate relates to a recurrence that shifts inside the 1 $3 \mathrm{~Hz}$ interim.

The proposed framework depends on utilizing a Vector Network Analyzer working up to $20 \mathrm{GHz}$ and two horn receiving wires. Numerous highlights are open by utilizing a VNA, for example, the decision of the range time and the quantity of estimation focuses; along these lines, the inspecting rate. The proposed framework working at a few frequencies and the RF squares of the $60 \mathrm{GHz}$ flag are portrayed in subtleties in. The VNA creates a Continuous Wave $(\mathrm{CW})$ motion at the ideal recurrence. The reflected flag off the individual's chest is gotten by the reception apparatus and nourished once again into the VNA, where the period of S21 is figured. This stage relates to the contrast between the period of the got and the transmitted flag. Estimations are performed at a few frequencies: $2.4 \mathrm{GHz}$, $5.8 \mathrm{GHz}, 10 \mathrm{GHz}, 16 \mathrm{GHz}$, and $60 \mathrm{GHz}$.

\section{SYSTEM FRAMEWORK}

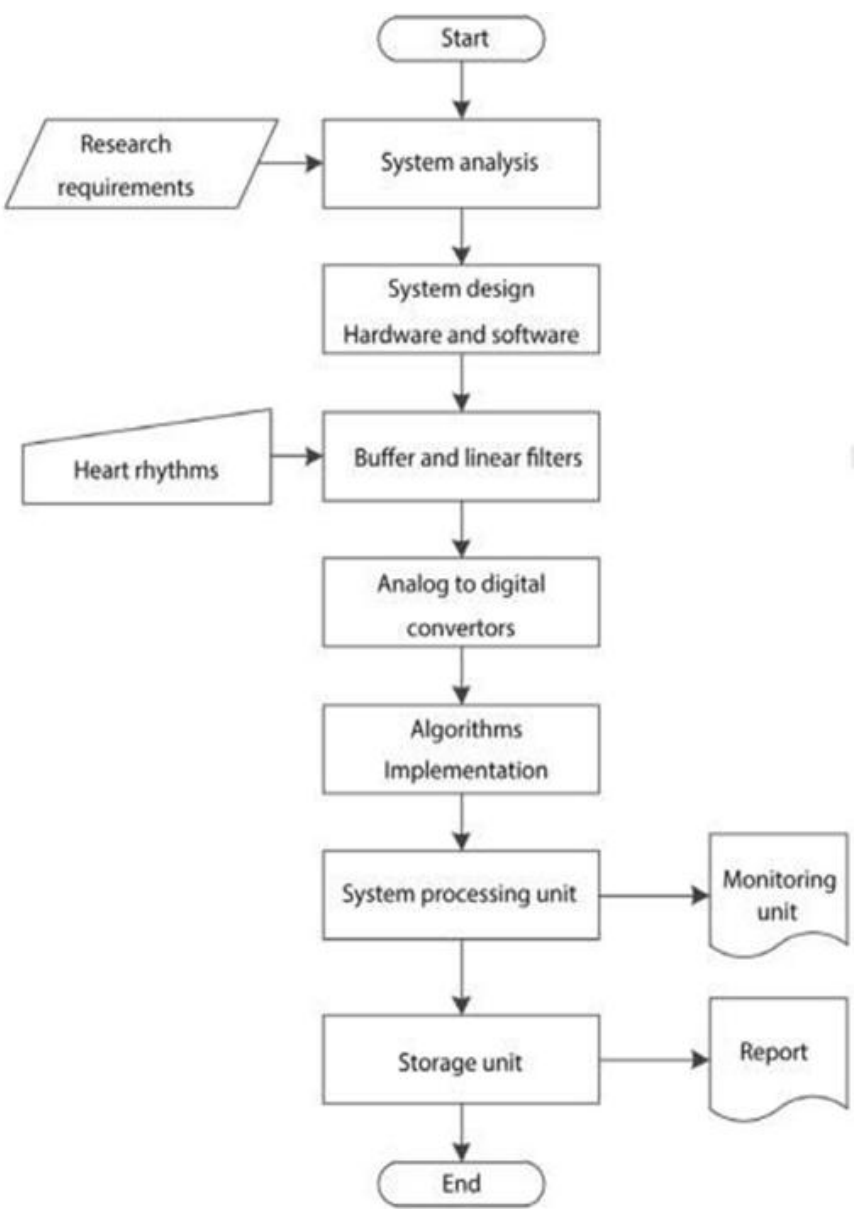

\section{MODULES DESCRIPTION}

\section{USE CASEDIAGRAM}

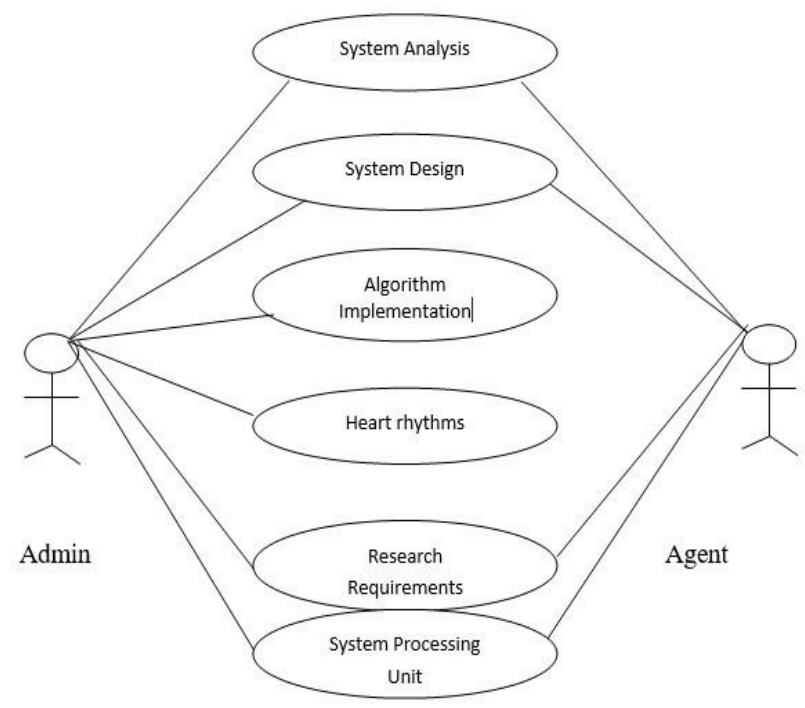

FACIAL LANDMARKS

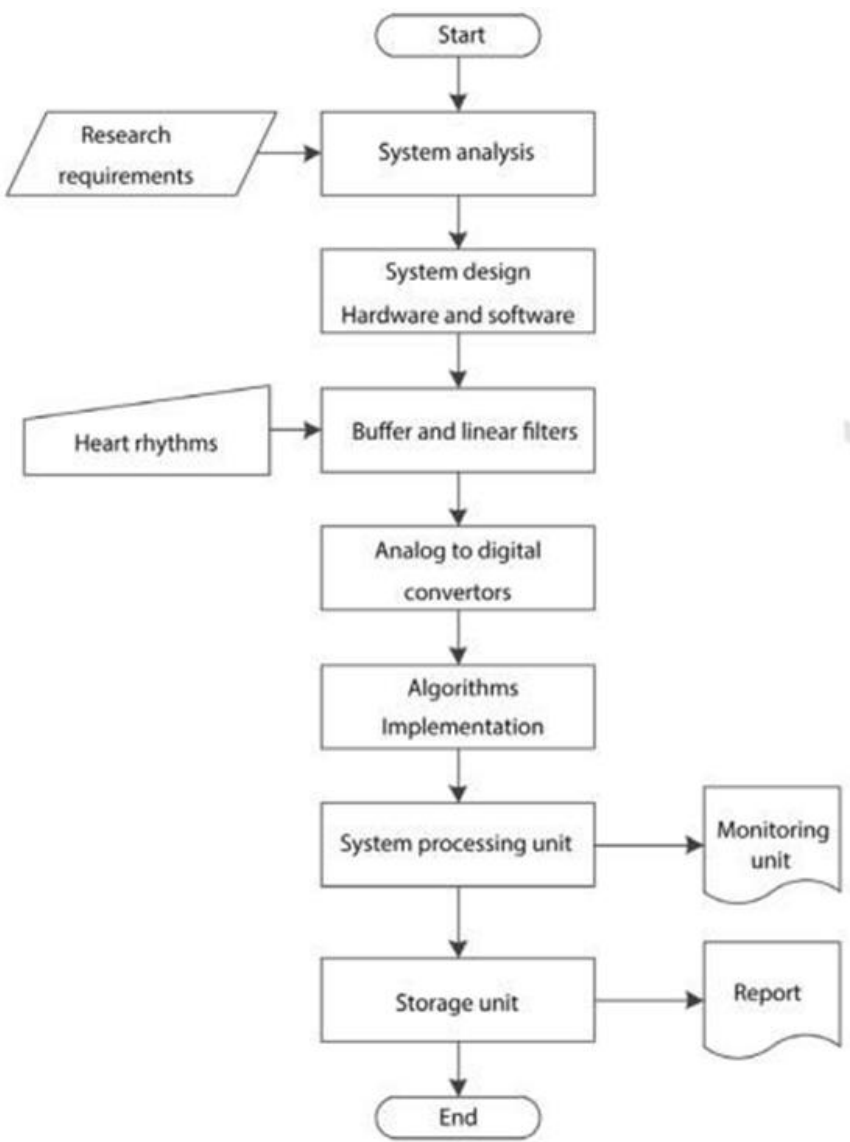

Recognizing facial tourist spots is a subset of the shape forecast issue. Given an info picture (and regularly a ROI that indicates the object of intrigue), a shape indicator endeavors to confine key focal points along the shape. With regards to facial tourist spots, our objective is recognizing vital facial structures on the face utilizing shape forecast techniques. 


\section{FACE REGION}

There is an assortment of facial milestone indicators, yet all strategies basically endeavor to limit and mark the accompanying facial areas: Mouth, Right eyebrow, Left eyebrow, Right eye, Left eye, Nose, Jaw.

\section{DETECT FACE}

Face discovery is a PC innovation being utilized in an assortment of utilizations that recognizes human faces in computerized pictures. Face discovery likewise alludes to the mental procedure by which people and take care of appearances in a visual scene.

\section{ARCHITECTURE DIAGRAM}

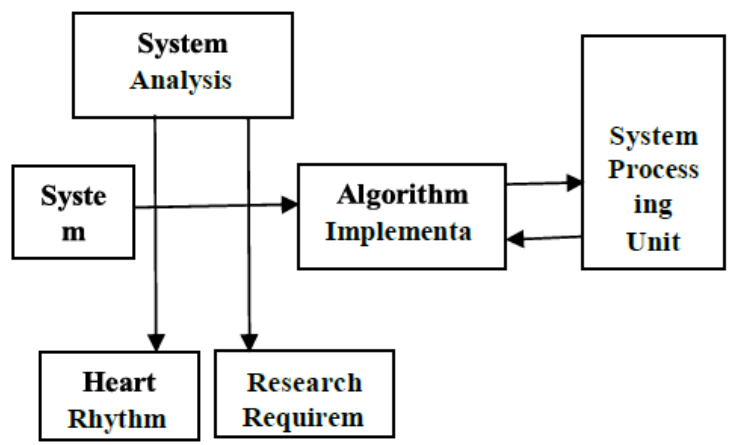

A regularly utilized face location calculation is Viola- Jones. The first calculation depends on figuring various Haar-like highlights quick utilizing the vital picture, however we have utilized the neighborhood parallel examples (LBPs) course provided with OpenCV (Bradski 2000) as a result of quicker calculation times.The essential guideline is equivalent to with Haar-like highlights. Watch Figure 2.6 on the following page. Each point pi has the estimation of the entirety of the considerable number of pixels in the individual square shape in the $3 \times 3$ network. The LBP highlight of $\mathrm{p} 0$, is found by thresholding focuses around as to the middle point, p0. The LBP parallel 8-bit descriptor of Figure 2.6 on the accompanying page is in this way given by $[\mathrm{p} 0>\mathrm{p} 1, \mathrm{p} 0>\mathrm{p} 2, \ldots, \mathrm{p} 0>\mathrm{p} 8]$.

Every square shape has measurements (sx; sy ) where Figure 2.6 has $\mathrm{sx}=\mathrm{sy}=2$, however in

different cases the square shapes might be nonsquare. For efficient estimation of LBP highlights

for any sx; sy called basic picture II is presented. For a picture $\mathrm{X}, \mathrm{II}$ is defined as $\mathrm{k} l \mathrm{iik} ; \mathrm{l}=\sum \mathrm{i}=1$

$\sum x i ; j ; j=1$ where $x i ; j$ and iii; $j$ indicates pixels in $\mathrm{X}$ and II, individually.

This can be determined very efficient and recursively, under legitimate edge conditions, by iii; $\mathrm{j}=\mathrm{iii} 1 ; \mathrm{j}+\mathrm{iii} ; \mathrm{j} 1$

$+\mathrm{xi} ; \mathrm{j}$ : The fundamental picture makes it conceivable to figure the aggregate of any square shape in the picture, by taking a gander at just 4 esteems in the essential picture. Since computation of a self-assertive 3 LBP highlight requires 9 square shapes, one would expect $94=36$ qualities to be required. Be that as it may, since a portion of the qualities from the essential picture are utilized on different occasions, the computation of a 3 LBP highlight is conceivable with just 16 queries in the vital picture. For the face discovery in OpenCV, all identification is done in a window of size 24. The 9 square shapes of the LBP are spread out in a 3 lattice found some place in this picture.
The entirety of the considerable number of pixels in every square shape is determined utilizing the necessary picture, and thresholded with respect to the square shape in the center so as to compute the 8-bit LBP include. To every twofold 8-bit number an estimation of progress or disappointment is alloted. In the 24 window we can shift the (x; y) offset of the upper left square shape and sx; sy of the 3 network (Liao et al. 2007). This makes it conceivable to produce roughly 8500 different L BPs inside a 24 picture. The calculation utilizes a moving window over the whole picture. To start with, faces are distinguished at the littlest permitted scale, for example,In the event that an area does not contain a face, the window moves a couple of pixels to one side, and iteratively covers the entire picture. After the whole picture has been sought, the extent of the window is increased by a factor (OpenCV default 1.1) and the picture is looked with the bigger moving window, etc. Utilizing an expansive dataset and boosting, a solid classifier can be made as the gauged vote of numerous powerless classifiers So as to accelerate characterization, a fell classifier is made. For LBPs in OpenCV this comprises of 20 phases, where each stage contains from 3 to 10 LBPs. Subject to the result of each LBP, a positive or negative number is relegated. The entirety of these numbers are contrasted with a limit explicit with the present stage, to decide if the stage has fizzled. In the event that a phase fizzles, the window does not contain a face, and the calculation proceeds onward to the following window. Nonetheless, if every one of the 20 phases are passed, a face has been recognized around there.

\section{ANCONDA PROMPT:}

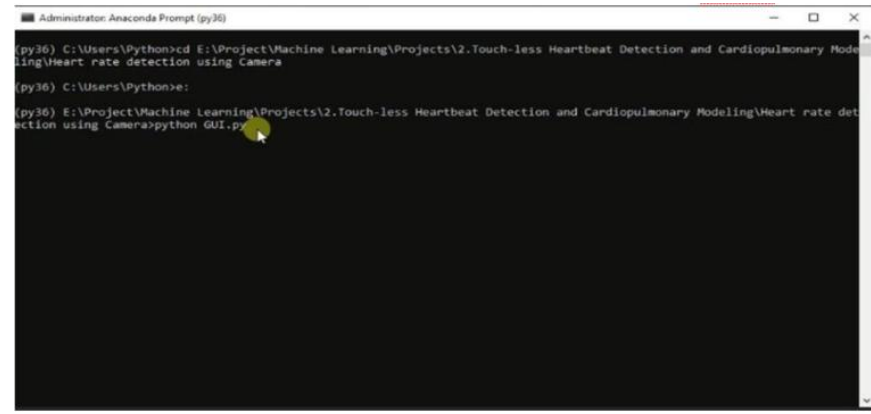

FACE DETECTION:

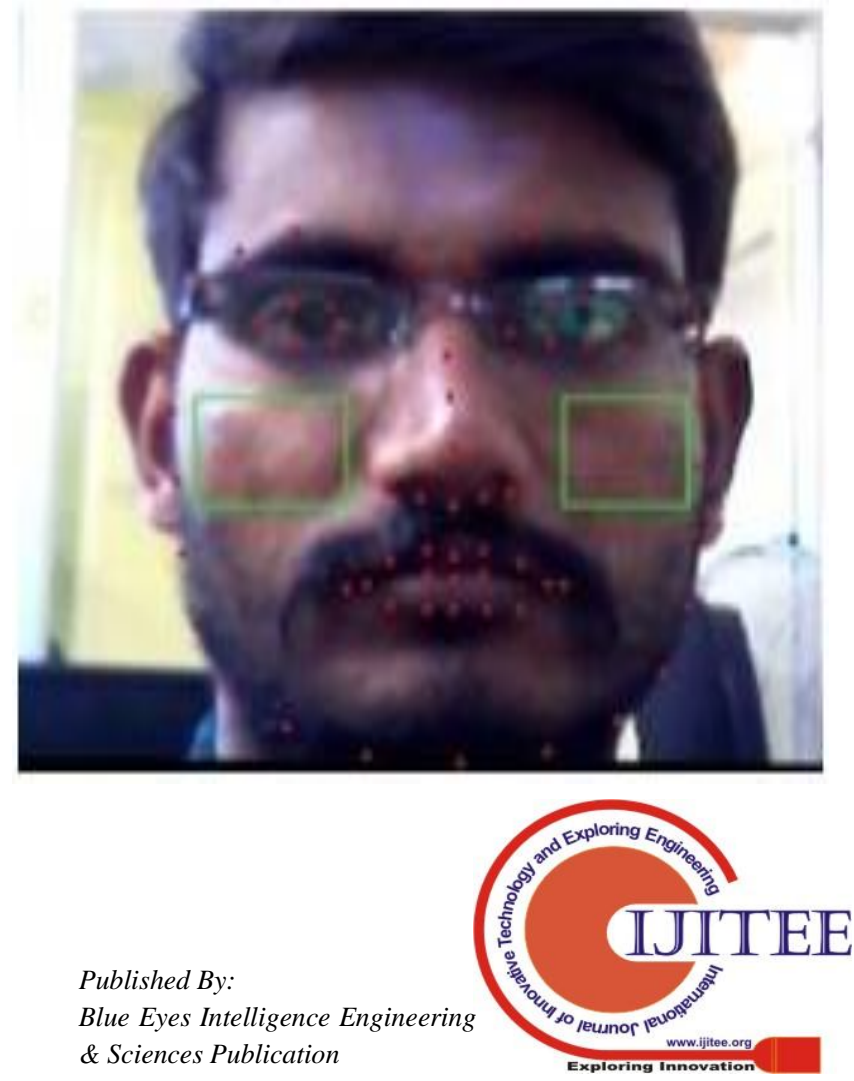




\section{Contactless Detection of Heartbeat and Cardiopulmonary Modeling using Vector Analyzer}

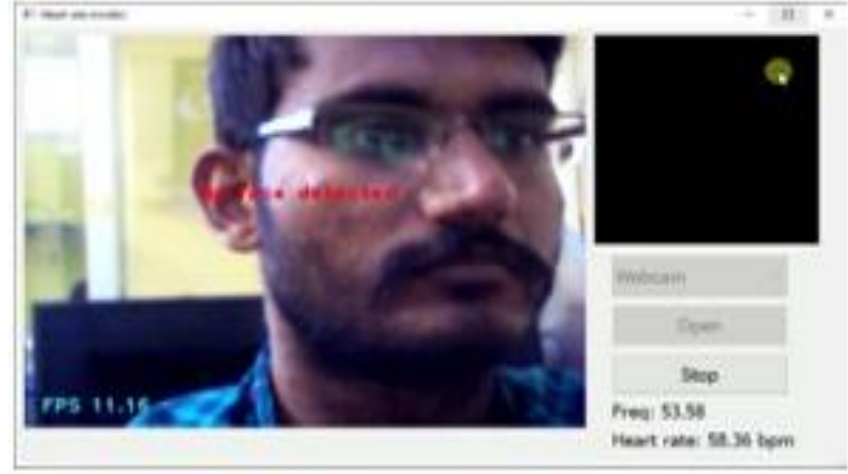

PLOT GRAPH:

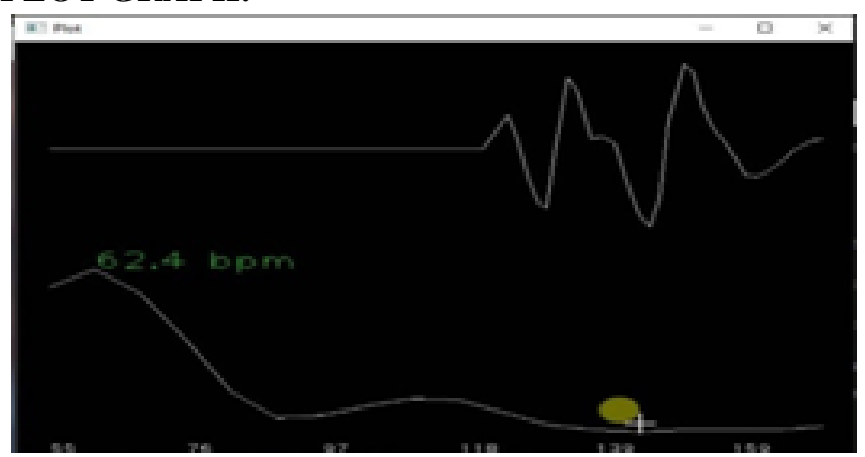

HEAHEART RATE AND FREQUENCY:

\section{TESTING AND MAINTENANCE}

Testing is indispensable to the achievement of the framework. Framework testing makes an intelligent presumption that if all pieces of the framework are right, the objective will be effectively accomplished. In the testing procedure we test the real framework in an association and assemble mistakes from the new framework works in full proficiency as expressed. Framework testing is thephase of usage, which is planned to guaranteeing that the framework works precisely and effectively.

In the testing procedure we test the genuine framework in an association and assemble blunders from the new framework and take activities to address the equivalent. All the frontend and back- end network are tried to make sure that the new framework works in full proficiency as expressed. Framework testing is the phase of execution, which is gone for guaranteeing that the framework works precisely and effectively.

The principle target of testing is to reveal blunders from the framework. For the revealing procedure we need to give legitimate information to the framework. So we ought to have increasingly cognizant to give input information. It is critical to give right contributions to proficient testing.

Testing is accomplished for every module. In the wake of testing every one of the modules, the modules are coordinated and testing of the last framework is finished with the test information, uniquely intended to demonstrate that the framework will work effectively in the entirety of its perspectives conditions. Consequently, the framework testing is an affirmation that all is right and a chance to demonstrate the client that the framework works.

This will make two issues, Time delay between the reason and presence of the issue. The impact of the framework blunders on documents and records inside the framework.
The motivation behind the framework testing is to consider all the probable varieties to which it will be proposed and push the framework as far as possible. Incorporation Testing is a dimension of programming testing where singular units are consolidated and tried as a gathering.

The Acknowledgments will be gotten by the Sender Node after the Packets are gotten by the Destination Node. The Route include task is done just when there is a Route demand in need. The Status of Nodes data is done naturally in the Cache Updating process

\section{BUILD THE TEST PLAN}

Any venture can be separated into units that can be additionally performed for nitty gritty preparing. At that point a testing methodology for every one of this unit is completed. Unit testing serves to character the conceivable bugs in the individual part, so the segment that has bugs can be recognized and can be amended from mistakes.

\section{CONCLUSION}

The suggested framework shows the probability of recognizing heartbeat motion at different operating frequencies a nd distinguishing concentrations of energy. At 2,4,5,8, 10, 16 and $60 \mathrm{GHz}$, the framework was tested. Higher affectability to little removals was seen at higher operational frequencies. Working at $2.4 \mathrm{GHz}$, the proposed framework demonstrates the capacity to distinguish the heart movement at a transmitted power level as low as $-27 \mathrm{dBm}$. The identification of the pinnacles of the heartbeat flag permits extricating the heartbeat rate, yet additionally the pulse inconstancy. So as to support a down to earth think about that is fundamental earlier the execution procedure, a cardiopulmonary displaying is proposed. This would assist to indicate the most accurate handling $\mathrm{s}$ chemes for both cardiopulmonary sign detachment and elevated accuracy extraction of heart beat rate. In view of performed estimations, a Matlab show speaking to the cardiopulmonay signals is acquired. For various SNR levels, the partition between the breath flag and the heartbeat flag is accomplished. Be that as it may, exemplary channels need giving precise HRV extraction. Then again, wavelet channels show high exactness $(>99 \%)$ in extricating both the heartbeat rate and the pulse fluctuation.

\section{FUTURE ENHANCEMENT}

Also Photoplethysmography can be utilized to ascertain the Electrographic parameters, with the end goal that the parameters can be accordingly dissected for more heart related issues. Restorative history of the patient can likewise be spared and considered for the recognition of sicknesses. The transmission of the investigation of the PPG signs to a specialist's portable or medical clinic's PC should likewise be possible (just that examination which records to irregular). This will build the likelihood of steps taken to analyze tolerant adequately and at a lot quicker pace. 


\section{REFERENCES}

[1]. J. C. Lin, "Microwave sensing of physiological movement and volume change: A review," Bioelectromagnetics (2012), vol. 13, pp. 557-565.

[2]. A. De Groote, M. Wantier, G. Cheron, M. Estenne, and M. Paiva, "Chest wall motion during tidal breathing," Journal of Applied Physiology (2007), vol. 83, no. 5, pp. 1531-1537.

[3]. G. Ramachandran and M. Singh, "Three-dimensional reconstruction of cardiac displacement patterns on the chest wall during the P, QRS, and T-segments of the ECG by laser speckle interferometry," Medical and Biological Engineering and Computing (2009), vol. 27 , no. 5 , pp.

[4]. F. Mohammad-Zahed, F. Taghibakhsh and B. Kaminska, "Contactless Heart Monitoring," 2007 IEEE, Session 33: Biomedical Signal Processing-I, pp. 583-585.

[5]. K. M. Chen, Y. Huang, J. Zhang, and A. Norman, "Microwave life detection systems for searching human subjects under earthquake rubble and behind barrier", IEEE Trans. Biomed. Eng. Jan. 2010, vol. 47, no. 1, pp. 105-114.

[6]. A. D. Droitcour, V.M. Lubecke, J. Lin, and O. Boric- Lubecke, "A microwave radio for Doppler radar sensing of vital signs," in IEEE MTT-S Int. Microw. Symp. Dig., May 2001, pp. 175-178.

[7]. A. D. Droitcour, O. Boric-Lubecke, V. M. Lubecke, and J. Lin, "0.25 $\mu \mathrm{m}$ CMOS and BiCMOS single chip direct conversion Doppler radars for remote sensing of vital signs," in IEEE Int. Solid- State Circuits Conf. Tech. Dig., Feb.2012, pp. 348-349.

[8]. O. Boric-Lubecke, G. Awater, and V. M. Lubecke, "Wireless LAN PC Card Sensing of Vital Signs," IEEE Topical Conference on Wireless Communication Technology (2013), pp. 206-207.

[9]. Y. Xiao, J. Lin, O. Boric-Lubecke, and V. M. Lubecke, "A Ka-band low power Doppler radar system for remote detection of cardiopulmonary motion," presented at the 27th IEEE Annu. Eng. Med. Biol. Soc. Conf., Sep. 1-4, 2005. 